2. Manyon A, Shipengrover J, McGuigan D, Haggerty $M$, James $P$, Danzo A. Defining differences in the instructional styles of community preceptors. Fam Med. 2003;35:181-186.

3. Ricer RE, Filak AT, David AK. Determining the costs of a required third-year family medicine clerkship in an ambulatory setting. Acad Med. 1998;73:809-811.

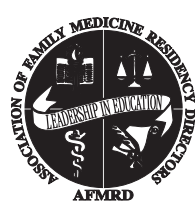

From the Association of Family Medicine Residency Directors

Ann Fam Med 2005;3:560-561. DOI: 10.1370/afm.410.

\section{SCHOLARLY ACTIVITY AND RESIDENCY TRAINING: SEEKING STRATEGIC PARTNERSHIPS}

The American Academy of Family Physicians (AAFP) and the Accreditation Council for Graduate Medical Education's (ACGME) program requirements for residency education in family practice acknowledge the importance of research and other scholarly activity in residency training. ${ }^{1}$ Included in the core competencies of medical knowledge, practice-based learning and improvement, and systems-based practice, the ACGME requires formal scholarly activity to occur in residency programs. While not directly stated, scholarly activity is often used as an umbrella term under which research is included as a separate entity.

While some research is often included in the residency curriculum, research is often but not consistently a required component of training. In a survey of family practice residency program directors, Neale ${ }^{2}$ found that $48.6 \%$ of responding programs required a resident research project. The top reasons for requiring resident research were to develop critical thinking and patient care skills and to promote an understanding of the medical literature. The top reasons for not requiring resident research were an attitude that it was not necessary and lack of faculty or time.

Overall, family medicine residency directors are supportive of scholarly activity in their programs. In a survey by DeHaven, ${ }^{3}$ more than one half of family medicine residency directors felt that their training program actively promotes research. Furthermore, 3 of 4 survey respondents indicated that involving residents in research was a goal of the program.

To successfully integrate research and scholarly activity and to overcome acknowledged barriers, residency programs require enthusiastic faculty that possess the skills, expertise, experience, and success in this area. Faculty involvement has been a reported char- acteristic of programs that are successful in research. ${ }^{1}$ Currently, only $12.9 \%$ of family practice residency programs require faculty to engage in research or scholarly activity. ${ }^{2}$ As such, program directors may need to seek assistance outside of their residencies in order to produce a successful scholarly activity curriculum.

In addition to promoting excellence in family medicine residency training, the Association of Family Medicine Residency Directors (AFMRD) and its Board of Directors is committed to the following goals:

- To represent family medicine residency program directors at a national level and provide a political voice for them in appropriate areas

- To develop the art and science of resident education in family medicine

- To improve the quality of education of family physicians

- To promote the ethical behavior in all aspects of residency operation

- To promote communication and cooperation between family medicine residency programs and other members of the family medicine family

- To provide a network for mutual assistance among family medicine residency directors

- To enhance the administrative operation of family medicine residencies

Consistent with these goals and to assist with overcoming acknowledged barriers, the AFMRD is committed to serving as a resource for residency directors in their efforts to incorporate formal scholarly activity curriculum into their residency programs. AFMRD seeks strategic partnerships with fellow family medicine organizations to assist in these efforts. For instance, the AFMRD will partner with the North American Primary Care Research Group (NAPCRG) to integrate both research and quality improvement as recognized scholarly activities and to develop a scholarly activity curricula that provides a structure as well as flexibility for program directors. In addition, the organization seeks to develop partnerships between NAPCRG members and specific residency programs to provide the research experience and expertise not always present in family medicine residency faculty members.

In terms of other key organizations, the AFMRD seeks the assistance of peer-reviewed publications such as the Annals of Family Medicine, Family Medicine, the Journal of the American Board of Family Medicine and others to actively seek, promote, and publish work produced by residency faculty and family medicine residents. In particular and as an encouragement to future researchers, the promotion and publication of the products of resident research should be a priority. As an example, these publications could sponsor a resident research competition and publish the results in a special edition 
as a means of stimulating resident research activities and fostering the development of our future family medicine scholars.

Through overcoming challenges and by seeking strategic partnerships, the AFMRD is actively pursuing many of its stated goals and central mission of serving as a resource for family medicine residency directors.

Peter J. Carek, MD, MS, FAAFP, Treasurer, AFMRD

David Araujo, MD, Member-at-Large, AFMRD

Peter M. Nalin, MD, FAAFP Immediate Past President, AFMRD

\section{References}

1. DeHaven MJ, Wilson GR, O'Connor-Kettlestrings P. Creating a research culture: what we can learn from residencies that are successful in research. Fam Med. 1998;30:501-517.

2. Neale AV. A national survey of research requirements for family practice residents and faculty. Fam Med. 2002;34:262-267.

3. DeHaven MJ, Wilson GR, Murphree DD, Grundig JP. Family practice residency directors' views on research. Fam Med. 1997;29:33-37.

$\begin{array}{ll}\text { NORTH } & \\ \text { AMERICAN } & \\ \text { PRIMARY CARE } & \text { From the North American } \\ \text { RESEARCH } & \text { Primary Care Research Group }\end{array}$

Ann Fam Med 2005;3:561-562. DOI: 10.1370/afm.411.

\section{UK LESSONS FOR US PRIMARY CARE}

Primary care is now acknowledged to be a foundation of effective, sustainable health care for populations, with favorable effects on access to care, comprehensiveness, continuity, efficiency, and equity. ${ }^{1}$ In addition, variation in health care arrangements and policies across nations presents opportunities to compare and learn across national boundaries about what is working and how well in primary care.

The relatively poor performance of the US health care system has provoked a willingness to change that in a well-performing system would probably not exist. ${ }^{2}$ Primary care physician offices in the United States are being redesigned somewhat haphazardly with numerous opportunities to improve and some opportunities to diminish the desired effects of primary care. The United Kingdom's primary care system, despite being strong, is also in the midst of dramatic changes orchestrated through the National Health Service. ${ }^{3}$ This combination in the United States and the United Kingdom is potent and presents immediate opportunities for decision makers in both countries to guide the "remake" of primary care with more real-world experience than is available in either nation alone.

In early June 2005 there was a face-to-face exchange visit between representatives of the Wash- ington, DC-based Robert Graham Center and the National Primary Care Research and Development Centre in Manchester, United Kingdom. Six examples of "30,000-foot level" conclusions reached on the basis of the specifics learned during the visit are: ${ }^{4}$

1. In both the United Kingdom and the United States, policy makers have begun to realize the great potential in primary care. However, the systems of care delivery and business plans for primary care are not adequate. Some revisions now underway may be converting the physician's role as a trusted personal physician doing hugely meaningful work, to a job-holder with a rulebook. The net impact of such changes may not be positive for physicians, patients, or health care systems.

2. Experiments in primary care delivery abound in both countries, and decisions are being made quickly, with little or no evaluation. Imbedding evaluations in new approaches/programs and responding to findings in nearly real time is possible in both countries. Sharing findings from real-time evaluations can also occur quickly, to mutual advantage.

3. No one knows how to structure practice and primary care physician compensation to incentivize and cover the full costs of robust primary care, but it is clear that further investments in primary care are necessary to garner its powerful, salutary effects for entire populations. The United Kingdom is making deliberate investments into its primary care infrastructure, while the United States seems to be bleeding revenue out of primary care while increasing its overhead. With an amazingly thin evidence base, both countries are pushing toward paying for performance improvements in practice, and this approach is having an effect on primary care practice-sometimes for the better. If some portion of primary care payment is based on performance measures, it will be necessary in the United States, as already done in the United Kingdom, to establish a denominator ( $\mathrm{eg}$, a register of patients for which the practice can be held accountable) for practices to use in assessing their performance.

4. Teamwork is no longer elective in primary care, but a huge gap still exists between the teamwork that is feasible through asynchronous, information technology-enabled care and what is currently happening. Many case studies are in play in both countries, under differing conditions. Training and educational strategies for teamwork among health professionals are lagging badly in both countries, and this is likely to emerge soon as a rate-limiting step in providing high-performance primary care.

5. Both countries are overconsuming international medical graduates from developing countries, probably to their own advantage but likely to the detriment of the donor nations. 